\title{
Dynamics of Uncertain Opinion Formation: An Agent-Based Simulation
}

\author{
Haiming Liang ${ }^{1,2}$, Yucheng Dong ${ }^{2}$, Cong-Cong $\mathbf{L i}^{2}$ \\ ${ }^{1}$ School of Economics \& Management, Xidian University, 266 Xinglong Section of Xifeng Road, Xi'an, Shaanxi \\ 710126, China \\ ${ }^{2}$ Business School, Sichuan University, No.24 South Section 1, Yihuan Road, Chengdu, 610065 China \\ Correspondence should be addressed to ycdong@scu.edu.cn
}

Journal of Artificial Societies and Social Simulation 19(4) 1, 2016

Doi: 10.18564/jasss.3111 Url: http://jasss.soc.surrey.ac.uk/19/4/1.html

Received: 19-05-2015 Accepted: 03-07-2016 Published: 31-10-2016

\begin{abstract}
Opinion formation describes the dynamics of opinions in a group of interaction agents and is a powerful tool for predicting the evolution and diffusion of the opinions. The existing opinion formation studies assume that the agents express their opinions by using the exact number, i.e., the exact opinions. However, when people express their opinions, sentiments, and support emotions regarding different issues, such as politics, products, and events, they often cannot provide the exact opinions but express uncertain opinions. Furthermore, due to the differences in culture backgrounds and characters of agents, people who encounter uncertain opinions often show different uncertainty tolerances. The goal of this study is to investigate the dynamics of uncertain opinion formation in the framework of bounded confidence. By taking different uncertain opinions and different uncertainty tolerances into account, we use an agent-based simulation to investigate the influences of uncertain opinions in opinion formation from two aspects: the ratios of the agents that express uncertain opinions and the widths of the uncertain opinions, and also provide the explanations of the observations obtained.
\end{abstract}

Keywords: Opinion formation, Uncertain opinions, Uncertainty tolerance, Communication regime, Agent-based simulation.

\section{Introduction}

1.1 Opinion formation is a powerful tool for predicting the evolutions and diffusion of opinions Afshar \& Asadpour 2010). Opinion formation describes the dynamics of opinions in a group of interaction agents (Urbig et al. 2008). There are two varieties of stabilized results (i.e., consensus and clusters) in opinion formation (Hegselmann et al. 2002). Many opinion formation models have been proposed to discuss the conditions of forming the stabilized results.

1.2 The study of opinion formation went back to (French Jr[1956). According to French's study, different types of studies on opinion formation have been proposed (Hegselmann et al. 2015): (i) opinion formation with continuous or discrete time (e.g., Lorenz 2005; Salzarulo 2006;|Blondel et al. 2009), (ii) opinion formation based on different communication regimes (e.g., DeGroot 1974||Latané|1981||Deffuant et al.|2000 Hegselmann et al.|2002, Krapivsky \& Redner|2003: Urbig et al. 2008 Deffuant et al. 2013||Lim et al. 2014), (iii) opinion formation with the multi-dimensional space of possible opinions (Laguna et al.|2003; Fortunato et al.2005: Etesami et al.2013), (iv) opinion formation in a specified network (Friedkin \& Johnsen|1990|| Weisbuch|2004|| Mckeown \& Sheehy 2006, Righi \& Carletti 2009: Wang \& Shang 2015), and (v) opinion formation considering noises (Pineda et al.2009, 2013).

1.3 The bounded confidence model assumes that each agent solely communicates with the agents who hold similar opinions and ignores the agents that have sufficiently different opinions. The earliest bounded confidence models have been introduced independently by Hegselmann and Krause (2002) and by Deffuant and Weisbuch (2000). The two bounded confidence models are called the HK model and the DW model, respectively. In the HK model, agents synchronously update their opinions by averaging all opinions in their confidence sets; in the 
DW model, agents follow a pairwise-sequential updating mechanism. Based on the HK and DW models, interesting extended research studies regarding the $\mathrm{HK}$ model and the DW model have been conducted (Fortunato et al. 2005, Ceragioli \& Frasca 2012; Morarescu \& Girard 2011).

1.4 Previous studies have significantly advanced the bounded confidence models. In this study, we propose the dynamics of uncertain opinion formation in the framework of bounded confidence. This study is motivated by the following aspects:

1. In the existing studies, the agents express opinions by using the exact number, i.e., the exact opinions. However, whether the opinion formation occurs in daily life or in the context of the Internet, the opinions of the agents often exhibit uncertainty. For example, when people express their opinions, sentiments, or support emotions regarding different issues, such as politics, products, and events, they often cannot provide exact opinions, but express uncertain opinions. Generally, the numerical intervals are the most basic formats of uncertain opinions (Dong et al. 2013; Dong \& Herrera-Viedma 2015). Thus, it is necessary to propose the uncertain opinion formation model, which will provide a foundation for investigating the dynamics of uncertain opinion formation.

2. In the practical opinion formation problem, the agents who encountered uncertain opinions often show different uncertainty tolerances. The differences in uncertainty tolerances are close to the culture backgrounds and characters of agents (Sutton et al.2004). For example, the agents with decisive or perfection seeking characters are interested in exact opinions, and they hope to communicate with the exact opinions. Thus, it is necessary to investigate the dynamics of uncertain opinion formation by considering different uncertainty tolerances.

1.5 The proposal can be applied to address certain opinion formation problems in the real world such as, when the government attempts to analyse the dynamics of public opinions on introducing the chemical project, some citizens may express uncertain opinions on the necessity of introducing the chemical project. Furthermore, different citizens who encountered uncertain opinions show different uncertainty tolerances. Therefore, when the proposal incorporated the above uncertainty factors, it can provide the decision support for the government to analyse the dynamics of public opinions.

1.6 The remainder of this study is organized as follows. Section 2 introduces the HK bounded confidence model. Then, Section 3 proposes the uncertain opinion formation model in the framework of bounded confidence. Next,Section 4 discuss the influences of uncertain opinions in opinion formation. In Section 5, the influences of uncertain tolerances in opinion formation are investigated. Finally, concluding remarks are included in Section 6 .

\section{The HK Bounded Confidence Model}

2.1 In this section, we briefly introduce the HK bounded confidence model, which is also the basic model of this study. The DW model and the HK model are very similar but differ mainly in the communication regime Urbig et al.2008). Thus, if we adopt the DW model as the basic model, a similar work will be conducted.

2.2 Consider an opinion formation problem. Let $A=\left\{A_{1}, A_{2}, \ldots, A_{N}\right\}$ be a set of agents, and $t$ be a discrete time, respectively. Let $X(t)=\left(x_{1}(t), x_{2}(t), \ldots, x_{N}(t)\right)^{T}$ be the opinion profile at time $t$, where $x_{i}(t) \in[0,1]$ denotes the exact opinion expressed by agent $A_{i} \in A$ at time $t$. Let $\varepsilon$ be the homogeneous bounded confidence of the agents.

2.3 Let $I\left(A_{i}, X(t)\right)$ be the confidence set of agent $A_{i}$ at time $t$, and let $w_{i j}(t)$ be the weight that agent $A_{i}$ assigns to agent $A_{j}$ at time $t$, i.e.,

$$
w_{i j}(t)=\left\{\begin{array}{ll}
\frac{1}{\# I\left(A_{i}, X(t)\right)}, & A_{j} \in I\left(A_{i}, X(t)\right) \\
0, & A_{j} \notin I\left(A_{i}, X(t)\right)
\end{array} \quad, \quad i=1,2, \ldots, N,\right.
$$

where $I\left(A_{i}, X(t)\right)=\left\{A_{j}|| x_{i}(t)-x_{j}(t) \mid \leq \varepsilon\right\}$, and \#I(Ai, $\left.X(t)\right)$ is the number of agents in the set $I\left(A_{i}, X(t)\right)$.

2.4 The opinion $x_{i}(t+1)$ is then calculated as:

$$
x_{i}(t+1)=\sum_{A_{j} \in I\left(A_{i}, X(t)\right)} w_{i j}(t) x_{j}(t), \quad i=1,2, \ldots, N .
$$




\section{The Uncertain Opinion Formation Model in the Framework of Bounded Confidence}

3.1 In this section, based on the original HK model, we propose the uncertain opinion formation model in the framework of bounded confidence. In the proposed uncertain opinion formation model, the agents express their opinions by either using the exact number (i.e., the exact opinions) or using the numerical intervals (i.e., the uncertain opinions). Let $\bar{X}(t)=\left(\bar{x}_{1}(t), \bar{x}_{2}(t), \ldots, \bar{x}_{N}(t)\right)^{T}$ be the opinion profile at time $t$, where $\bar{x}_{i}(t)=$ $\left[\bar{x}_{i}^{L}(t), \bar{x}_{i}^{U}(t)\right] \subseteq[0,1]\left(\bar{x}_{i}^{L}(t) \leq \bar{x}_{i}^{U}(t)\right.$, for $\left.i=1,2, \ldots, N\right)$ denotes the opinion of agent $A_{i}$ at time $t$. Specially, $\bar{x}_{i}^{U}(t)>\bar{x}_{i}^{L}(t)(i=1,2, \ldots, N)$ indicates that agent $A_{i}$ expresses the uncertain opinion at time $t$.

3.2 The proposed uncertain opinion model consists of three steps. The first step is to determine the confidence set for each agent. Let $\tilde{I}\left(A_{i}, \bar{X}(t)\right)$ be the confidence set of $A_{i}$ at time $t$. Inspired by the original HK model, $\tilde{I}\left(A_{i}, \bar{X}(t)\right)$ is determined by

$$
\tilde{I}\left(A_{i}, \bar{X}(t)\right)=\left\{A_{j} \mid d_{i j}(t) \leq \varepsilon\right\}, \quad i=1,2, \ldots, N
$$

where $d_{i j}(t)$ denotes the Euclidean distance between the uncertain opinions $\bar{x}_{i}(t)$ and $\bar{x}_{j}(t)$, i.e.,

$$
d_{i j}(t)=\sqrt{\frac{1}{2}\left[\left(\bar{x}_{i}^{L}(t)-\bar{x}_{j}^{L}(t)\right)^{2}+\left(\bar{x}_{i}^{U}(t)-\bar{x}_{j}^{U}(t)\right)^{2}\right]}
$$

3.3 Then, the second step is to determine the weights that one agent assigns to other agents. Let $w_{i j}(t)$ be the weight that agent $A_{i}$ assigns to agent $A_{j}$ at time $t$, and $w_{i j}(t)$ is given by

$$
w_{i j}(t)=\left\{\begin{array}{ll}
\frac{1}{\# \tilde{I}\left(A_{i}, \bar{X}(t)\right)}, & A_{j} \in \tilde{I}\left(A_{i}, \bar{X}(t)\right) \\
0, & A_{j} \notin \tilde{I}\left(A_{i}, \bar{X}(t)\right)
\end{array}, \quad i=1,2, \ldots, N\right.
$$

where $\# \tilde{I}\left(A_{i}, \bar{X}(t)\right)$ is the number of agents in the set $\tilde{I}\left(A_{i}, \bar{X}(t)\right)$.

3.4 Finally, the third step is to determine the updated opinions for each agent. Due to the differences in culture backgrounds and characters of agents, people who encounter uncertain opinions often show different uncertainty tolerances. So, in this study the agents are divided into two types: the agents with the uncertainty tolerances, and the agents without the uncertainty tolerances. The agents with the uncertainty tolerances refer to the agents who can directly communicate both the exact opinions and uncertain opinions. The agents without the uncertainty tolerances refer to the agents who only communicate with exact opinions. Thus, when confronting uncertain opinions, the agents without the uncertainty tolerances will provide accurate estimations of the uncertain opinions. For notational simplicity, let $A^{u}$ be the set of agents with the uncertainty tolerances, and let $A^{o}$ be the set of agents without the uncertainty tolerances, where $A^{u} \cup A^{o}=A$ and $A^{u} \bigcap A^{o}=\emptyset$.

3.5 Specifically, let the agent $A_{i} \in A^{u}$, and $A_{j} \in \tilde{I}\left(A_{i}, \bar{X}(t)\right)$. Then $A_{i}$ will directly update his/her opinion based on the opinion $\left[\bar{x}_{j}^{L}(t), \bar{x}_{j}^{U}(t)\right]$. Let $\bar{x}_{i}(t+1)=\left[\bar{x}_{i}^{L}(t+1), \bar{x}_{i}^{U}(t+1)\right]$ be the opinions of agent $A_{i}$ at time $t+1$, then

$$
\begin{array}{ll}
\bar{x}_{i}^{L}(t+1)=w_{i i}(t) \bar{x}_{i}^{L}(t)+\sum_{A_{j} \in \tilde{I}\left(A_{i}, \bar{X}(t)\right), j \neq i} w_{i j}(t) \bar{x}_{j}^{L}(t), & A_{i} \in A^{u}, \\
\bar{x}_{i}^{U}(t+1)=w_{i i}(t) \bar{x}_{i}^{U}(t)+\sum_{A_{j} \in \tilde{I}\left(A_{i}, \bar{X}(t)\right), j \neq i} w_{i j}(t) \bar{x}_{j}^{U}(t), & A_{i} \in A^{u} .
\end{array}
$$

3.6 Let the agent $A_{i} \in A^{o}$ and $A_{j} \in \tilde{I}\left(A_{i}, \bar{X}(t)\right)$. When confronting the uncertain opinion $\left[\bar{x}_{j}^{L}(t), \bar{x}_{j}^{U}(t)\right]$ (i.e., $\left.\bar{x}_{j}^{L}(t)<\bar{x}_{j}^{U}(t)\right)$, the agent $A_{i}$ will provide the accurate estimation $f_{i j}(t)$ as the opinion of $A_{j}$, where $f_{i j}(t) \in$ $\left[\bar{x}_{j}^{L}(t), \bar{x}_{j}^{U}(t)\right]$. Notably, the accurate estimation $f_{i j}(t)$ in our simulation is randomly and uniformly selected from the uncertain opinion $\left[\bar{x}_{j}^{L}(t), \bar{x}_{j}^{U}(t)\right]$. Next, $A_{i}$ will update his/her opinion based on the accurate estimation $f_{i j}(t)$.

3.7 Let $\bar{x}_{i}(t+1)=\left[\bar{x}_{i}^{L}(t+1), \bar{x}_{i}^{U}(t+1)\right]$ be as before, then

$$
\begin{array}{ll}
\bar{x}_{i}^{L}(t+1)=w_{i i}(t) \bar{x}_{i}^{L}(t)+\sum_{A_{j} \in \tilde{I}\left(A_{i}, \bar{X}(t)\right), j \neq i} w_{i j}(t) f_{i j}(t), & A_{i} \in A^{o}, \\
\bar{x}_{i}^{U}(t+1)=w_{i i}(t) \bar{x}_{i}^{U}(t)+\sum_{A_{j} \in \tilde{I}\left(A_{i}, \bar{X}(t)\right), j \neq i} w_{i j}(t) f_{i j}(t), & A_{i} \in A^{o} .
\end{array}
$$


In the Appendix A, we compare the HK model with the proposed model, and analyze the proposed model to illustrate certain desired properties.

\section{The Influences of Uncertain Opinions in the Opinion Formation}

4.1 In this section, we assume that all the agents are with the uncertainty tolerances, i.e., $A^{u}=A$. We then investigate the influences of uncertain opinions in the opinion formation from two aspects: (1) the influences of the ratios of the agents expressing uncertain opinions, and (2) the influences of the widths of uncertain opinions. We consider four indexes in the investigation as follows:

(i) The number of clusters $(N C) . N C$ is the number of different opinions in the stabilized results. Larger $N C$ values indicate more different opinions among the agents in the stabilized results. In particular, $N C=1$ represents that the opinions of agents reach a consensus.

(ii) The ratios of the extremely small clusters in all clusters $\left(r_{E S C}\right)$. Extremely small cluster $(E S C)$ is the cluster which includes a few of agents. Let $S=\left\{s_{1}, s_{2}, \cdots, s_{N C}\right\}$ be the set of clusters in the stabilized results, where $s_{v}$ denotes the $v$ th cluster, $v=1,2, \ldots, N C$. Let $\# s_{v}$ be the number of agents in the cluster $s_{v}$. Based on the study (Urbig et al. 2008), if \# $s_{v} \leq \frac{\varepsilon N}{2}$, then $s_{v}$ is an extremely small cluster. Let \#ESC be the number of the extremely small clusters in the stabilized results, and let $r_{E S C}=\frac{\# E S C}{N C}$.

(iii) The ratios of the agents expressing the uncertain opinions in the stabilized results $\left(r_{s}\right)$. Assume that the stabilized results are formed at time $t$. Let $U O=\left\{A_{i} \mid x_{i}^{U}(t)>x_{i}^{L}(t), i=1,2, \ldots, N\right\}$ be the set of the agents expressing the uncertain opinions at time $t$. And let $\# U O$ be the number of agents in the set $U O$, and let $r_{s}=\frac{\# U O}{N}$.

(iv) The average widths of uncertain opinions in the stabilized results $(W U)$. Assume that the stabilized results are formed at time $t$. Let $U O$ and $\# U O$ be as before. Then, $W U$ is determined by

$$
W U=\frac{1}{\# U O} \sum_{A_{i} \in U O}\left(x_{i}^{U}(t)-x_{i}^{L}(t)\right) .
$$

4.2 Before investigating the influences noted above, we define certain variables in the simulation. Let $r \in[0,1]$ be the ratio of the agents expressing uncertain opinions. Let $\lambda \in[0,1]$ be the maximum width among all of the initial opinions, i.e., $\lambda=\max \left\{x_{i}^{U}(0)-x_{i}^{L}(0), i=1,2, \ldots, N\right\}$. Let $\lambda^{\prime} \in[0,1]$ be the average widths among all of the initial opinions, i.e., $\lambda^{\prime}=\sum_{i=1}^{N}\left(x_{i}^{U}(0)-x_{i}^{L}(0)\right) / N$.

\section{The influences of the ratios of the agents expressing uncertain opinions}

4.3 We investigate the influences of the ratios of the agents expressing uncertain opinions based on three criteria $N C, r_{E S C}$ and $r_{s}$. In the simulation, let $N=500$ and $\lambda=1$. The initial opinions $X(0)$ of $N$ agents are randomly and uniformly generated. Specifically, without loss of generality, we assume that the former $N \times r$ agents $A_{i}(i=1,2, \ldots, N \times r)$ express uncertain opinions, and the latter $N \times(1-r)$ agents $(i=N \times$ $r+1, N \times r+2, \ldots, N)$ express the exact opinions. Then, for the former $N \times r$ agents $A_{i}$, their uncertain opinions $\bar{x}_{i}(0)(i=1,2, \ldots, N \times r)$ are the subintervals that are randomly selected from [0,1], and for the latter $N \times(1-r)$ agents, their opinions $\bar{x}_{i}(0)(i=N \times r+1, N \times r+2, \ldots, N)$ are the exact numbers that are randomly selected from [0,1]. Next, using Eqs. (6)-(7) proceeds with the evolution of opinions. We set different $r$ and $\varepsilon$ values and run the simulation 1000 times, obtaining the average $N C, r_{E S C}$ and $r_{s}$ values under different parameters, which are shown in Figures 1-3.

4.4 Figure 1 shows two findings: (i) The average $N C$ values increase as $r$ increases and (ii) the average $N C$ values decrease as $\varepsilon$ increases. This implies that the larger ratios of the agents expressing the uncertain opinions will lead to more clusters. The opposite results will be obtained with the increase in the bounded confidences. These two observations from Figure 1 can be explained as follows:

In the simulation, we find that with the increase in the ratios of the agents expressing the uncertain opinions and the decrease in the bounded confidences, the interactions among the agents will decrease. As a result, more clusters will appear.

4.5 Figure 2 shows two findings: (i) The average $r_{E S C}$ values increase as $r$ increases, and (ii) The average $r_{E S C}$ values decrease as $\varepsilon$ increases. This implies that the ratios of the extremely small clusters in all clusters will increase with the increase in the ratios of the agents expressing the uncertain opinions. The opposite result will 


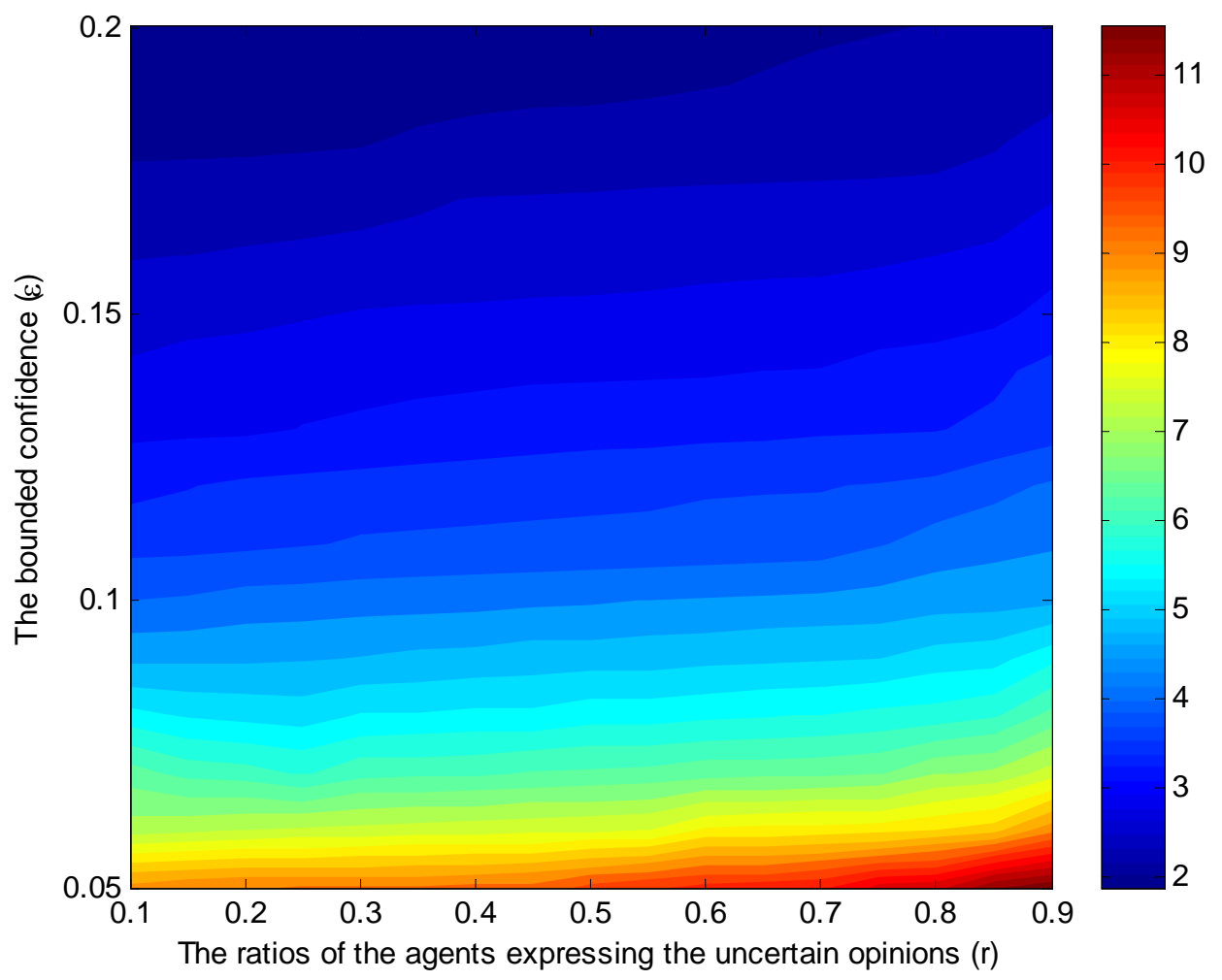

Figure 1: The average $N C$ values under different $r$ and $\varepsilon$ values

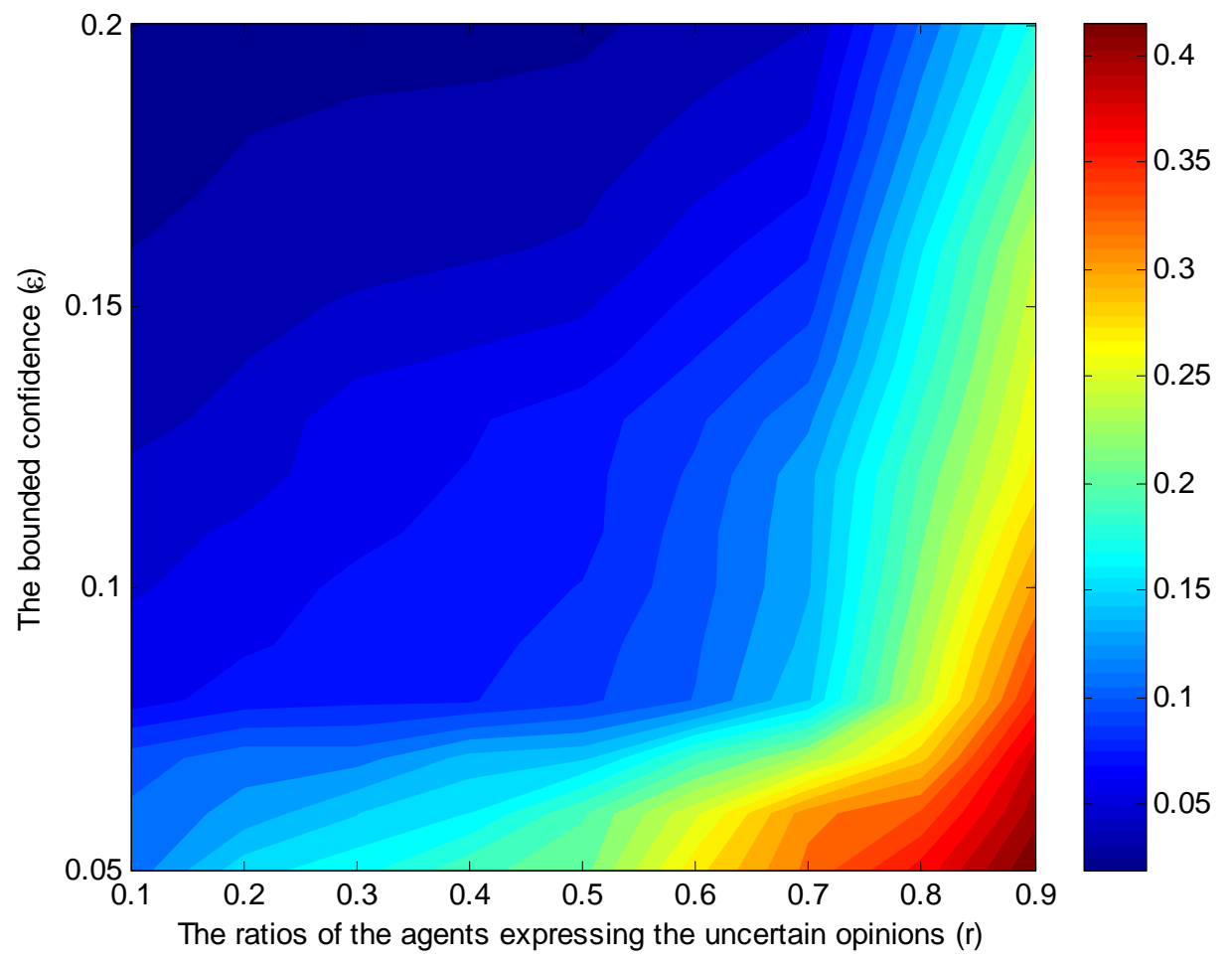

Figure 2: The average $r_{E S C}$ values under different $r$ and $\varepsilon$ values

be obtained with the increase in the bounded confidences. These two observations can be explained as follows: In the simulation, we find that the number of the isolated opinions at $t=0$ will increase with the increase in the ratios of the agents expressing the uncertain opinions and the decrease in the bounded confidences. Consequently, the larger ratios of the extremely small clusters in all clusters will appear. 


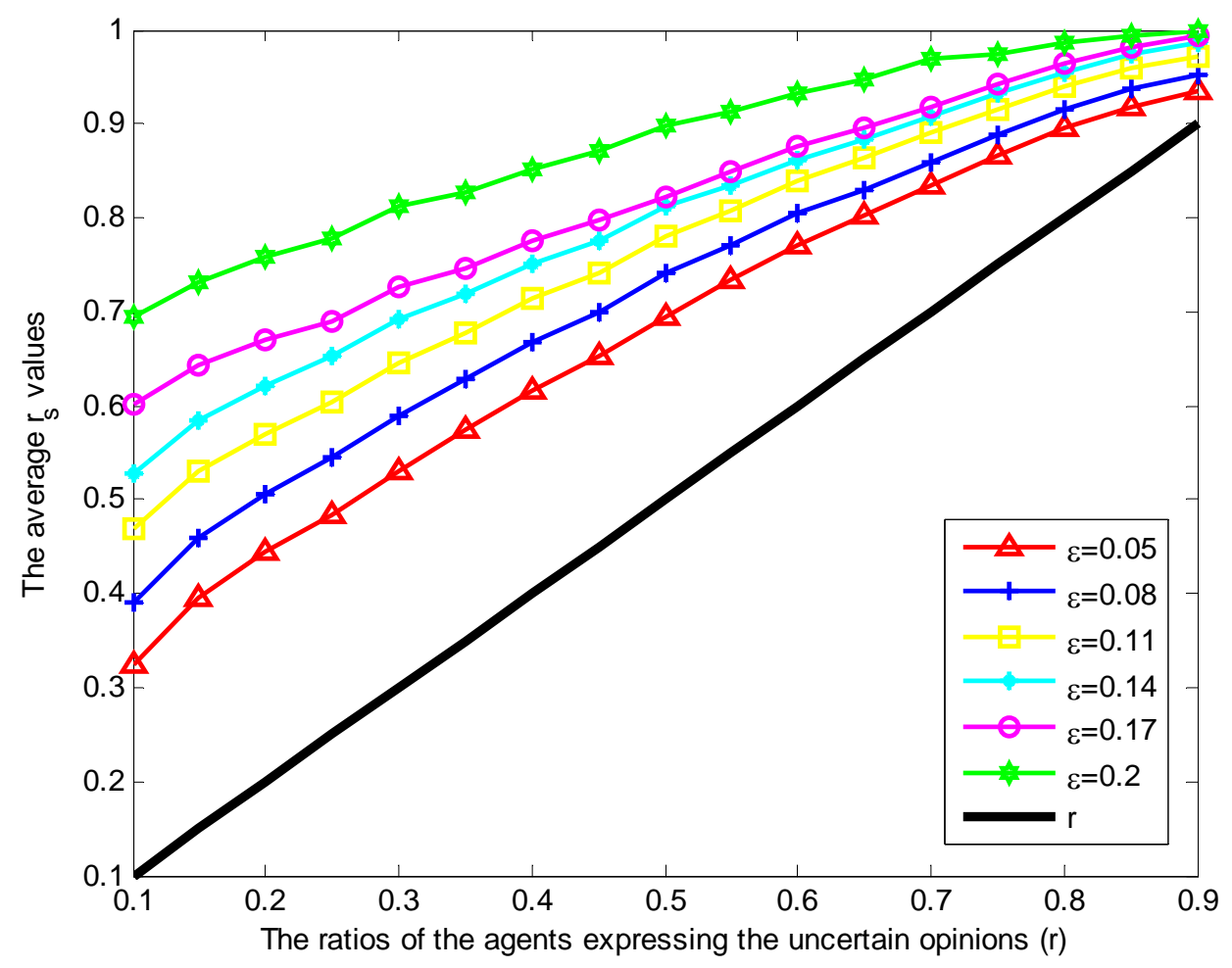

Figure 3: The average $r_{s}$ values under different $r$ and $\varepsilon$ values

4.6 Figure 3 shows two findings: (i) The average $r_{s}$ values increase as both $r$ and $\varepsilon$ increase, and (ii) The average $r_{s}$ values are larger than the $r$ values. This implies that with the increase in the ratios of the agents expressing the uncertain opinions and the bounded confidences, the ratios of the agents expressing the uncertain opinions in the stabilized result will increase. Meanwhile, the ratios of the agents expressing the uncertain opinions in the stabilized result will be larger than those in the initial time. These two observations can be explained as follows: It is clear that the larger ratios of the agents expressing the uncertain opinions in the initial time will lead to the larger ratios of the agents expressing uncertain opinions in the stabilized result. Meanwhile, when interacting with the uncertain opinions, the exact opinions in the initial time will gradually become uncertain. Consequently, the larger ratios of the agents expressing the uncertain opinions in the stabilized result will be yielded.

\section{The influences of the widths of uncertain opinions}

4.7 We investigate the influences of the width of uncertain opinions based on three criteria $N C, r_{E S C}$ and $W U$. In the simulation, let $N=500$ and $r=1$. We uniformly and randomly generate $N$ exact numbers $y_{i}(0)$ $(i=1,2, \ldots, N)$ in $[0,1]$. Based on the generated exact number, we randomly generate the uncertain opinions $\bar{x}_{i}(0)=\left[\bar{x}_{i}^{L}(0), \bar{x}_{i}^{U}(0)\right](i=1,2, \ldots, N)$, where $\bar{x}_{i}^{L}(0)=\max \left\{0, y_{i}(0)-\delta_{i} / 2\right\}$ and $\bar{x}_{i}^{U}(0)=\min \left\{y_{i}(0)+\right.$ $\left.\delta_{i} / 2,1\right\}$, and $\delta_{i}(i=1,2, \ldots, N)$ is the exact number that is randomly selected from $[0, \lambda]$. Clearly, the widths of all of the generated uncertain opinions are smaller than $\lambda$. Furthermore, the larger $\lambda$ values indicate that all of the generated opinions are with the larger average widths. Next, using Eqs. (6) and (7) proceeds with the evolution of opinions. We set different $\lambda$ and $\varepsilon$ values and run each simulation 1000 times, obtaining the average $N C, r_{E S C}$ and $W U$ values under different parameters, which are shown in Figures 4-6.

4.8 Figure 4 shows the following finding: The average $N C$ values increase as $\lambda$ increases. This implies that the number of clusters will increase with the increase in the widths of uncertain opinions.

4.9 Figure 5 shows the following finding: The average $r_{E S C}$ values increase as $\lambda$ increases. This implies that the ratios of the extremely small clusters in all clusters will increase with the increase in the widths of uncertain opinions. The findings observed in Figures 4 and 5 can be explained as follows: In the simulation, we find that with the increase in the widths of uncertain opinions, the interactions among the agents will decrease, and the 


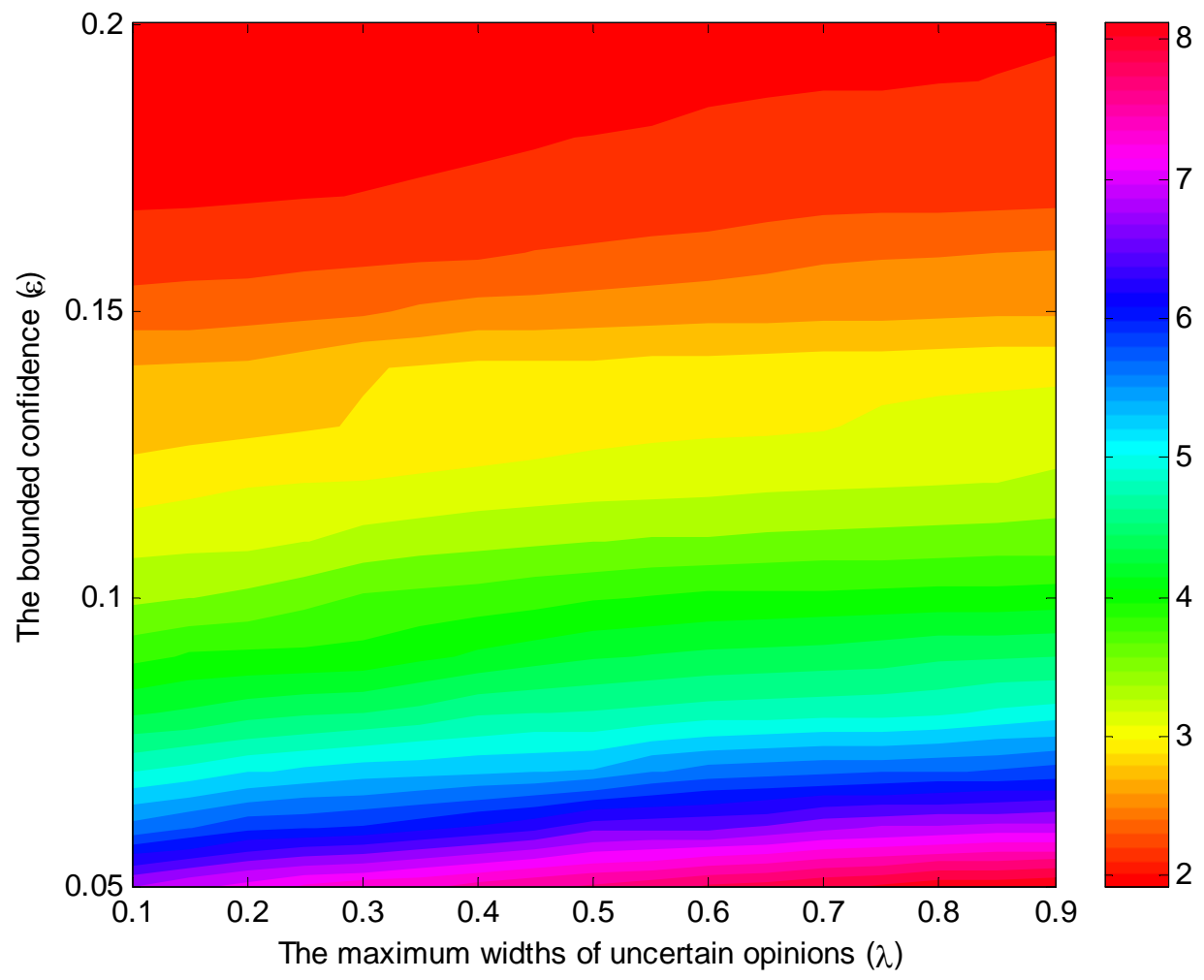

Figure 4: The average $N C$ values under different $\lambda$ and $\varepsilon$ values

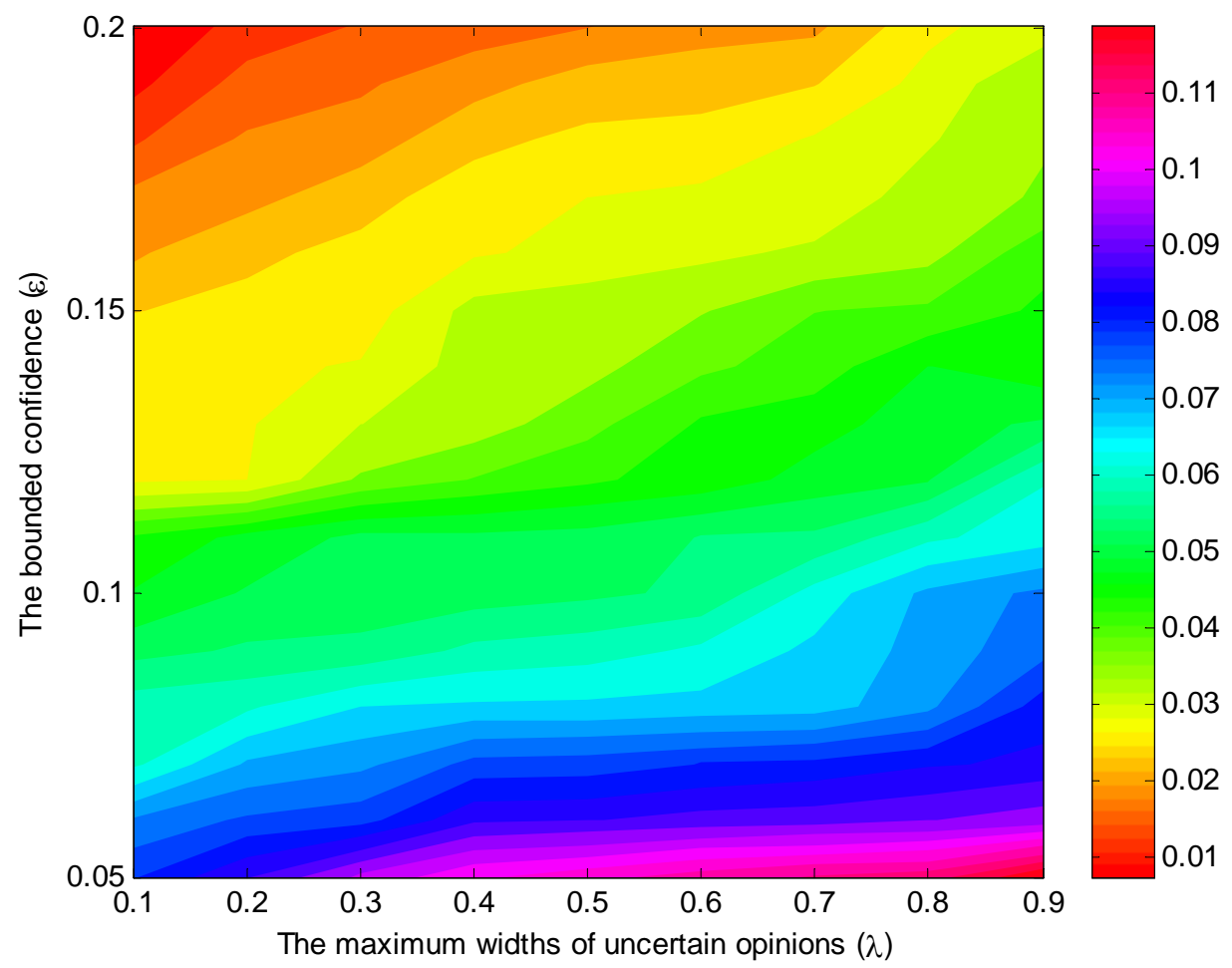

Figure 5: The average $r_{E S C}$ values under different $\lambda$ and $\varepsilon$ values

number of the isolated opinions will increase. As a result, more clusters and larger ratios of the extremely small clusters in all clusters will appear.

4.10 Figure 6 shows the following findings: (i) The average $W U$ values increase as $\lambda$ increases, and (ii) The average 


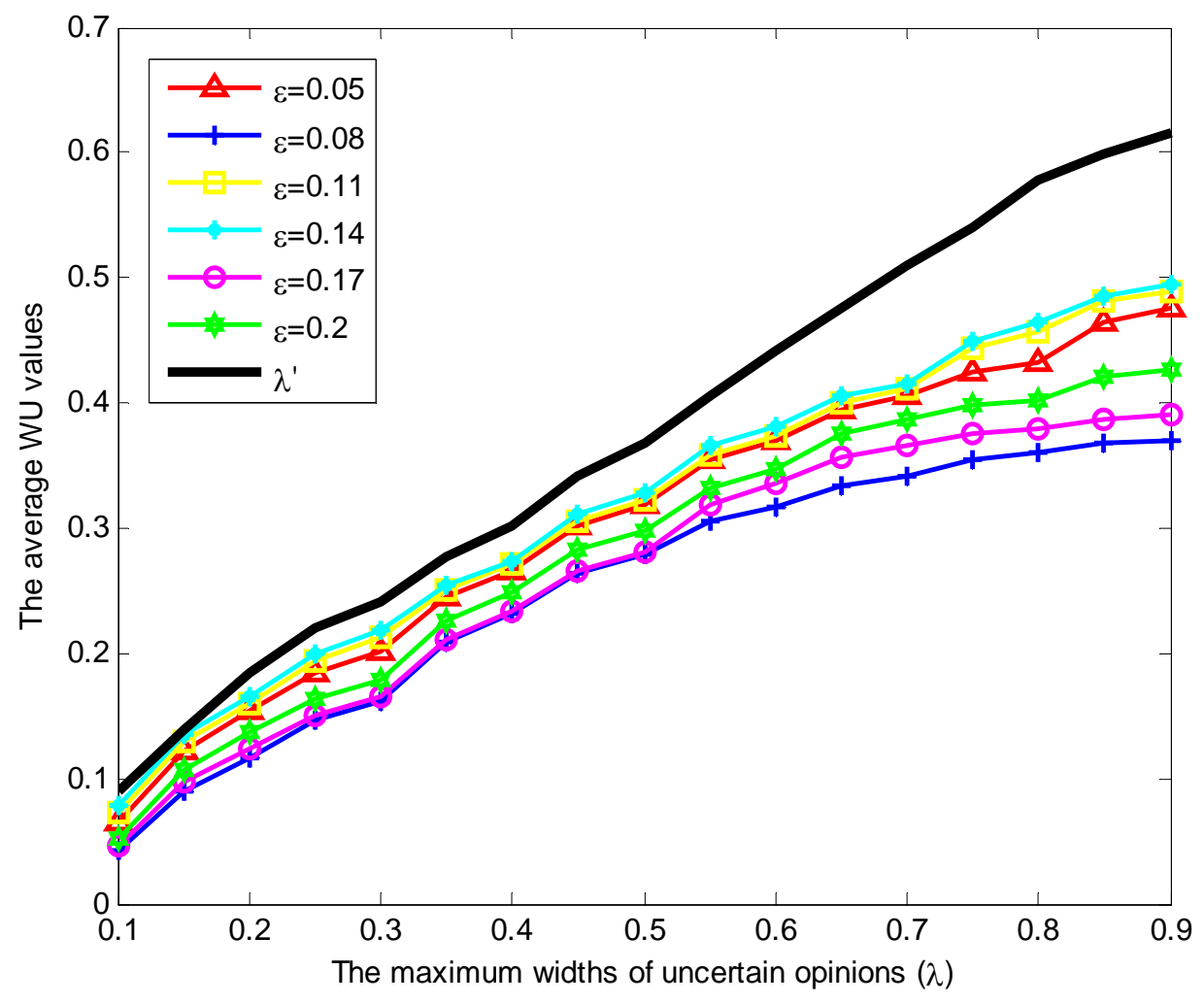

Figure 6: The average $W U$ values under different $\lambda$ and $\varepsilon$ values

$W U$ values are smaller than the $\lambda^{\prime}$ values. This implies that the widths of uncertain opinions in the stabilized results will increase with the increase in the widths of uncertain opinions in the initial time. Meanwhile, the average widths of uncertain opinions in the stabilized result will be smaller than those in the initial time. It is clear that the larger widths of uncertain opinions in the initial time will lead to the larger widths of uncertain opinions in the stabilized result. Meanwhile, in the simulation, we find that the number of uncertain opinions with the smaller widths will increase in the dynamics of uncertain opinion formation. Consequently, the average widths of uncertain opinions in the stabilized result will become smaller.

\section{The Influences of the Ratios of the Agents with the Uncertainty Tolerances}

5.1 We investigate the influences of the ratios of the agents with the uncertainty tolerances based on four criteria (i.e., $N C, r_{E S C}, r_{s}$ and $W U$ ) mentioned in section 4.1. Before investigating these influences, we denote the ratio of the agents with the uncertainty tolerances as $\beta$, where $\beta=\# A^{u} / A$ and $\beta \in[0,1]$. Let the agent $A_{i} \in A^{o}$ and $A_{j} \in \tilde{I}\left(A_{i}, \bar{X}(t)\right)$. When confronting the uncertain opinion $\left[\bar{x}_{j}^{L}(t), \bar{x}_{j}^{U}(t)\right]\left(\bar{x}_{j}^{L}(t)<\bar{x}_{j}^{U}(t)\right)$, the agent $A_{i}$ will provide the accurate estimation $f_{i j}(t)$ as the opinion of $A_{j}$, where $f_{i j}(t) \in\left[\bar{x}_{j}^{L}(t), \bar{x}_{j}^{U}(t)\right]$. Notably, the accurate estimation $f_{i j}(t)$ in our simulation is randomly and uniformly selected from the uncertain opinion $\left[\bar{x}_{j}^{L}(t), \bar{x}_{j}^{U}(t)\right]$.

5.2 In the simulation, we randomly selected $N \times \beta$ agents from $A$, and assume that these selected $N \times \beta$ agents are with the uncertainty tolerances, i.e., the selected $N \times \beta$ agents belong to the set $A^{u}$ and the other agents belong to the set $A^{o}$. If the agent $A_{i} \in A^{o}$ and $A_{j} \in \tilde{I}\left(A_{i}, \bar{X}(t)\right)$, the agent $A_{i}$ will provide the accurate estimation $f_{i j}(t)$ as the opinion of $A_{j}$, where $f_{i j}(t)$ is randomly and uniformly generated from $\left[\bar{x}_{j}^{L}(t), \bar{x}_{j}^{U}(t)\right]$. Next, we use the methods in sections 4.3 and 4.4 to generate the initial opinions $\bar{X}(0)$, and use Eqs. (6)-(9) to calculate the $N C, r_{E S C}, r_{s}$ and $W U$ values. Finally, we set $\varepsilon=0.1$ and different $\beta, r$ and $\lambda$ values, and run the simulation 1000 times, obtaining the average $N C, r_{E S C}, r_{s}$ and $W U$ values under different parameters, which are shown in Figures 7-9. 


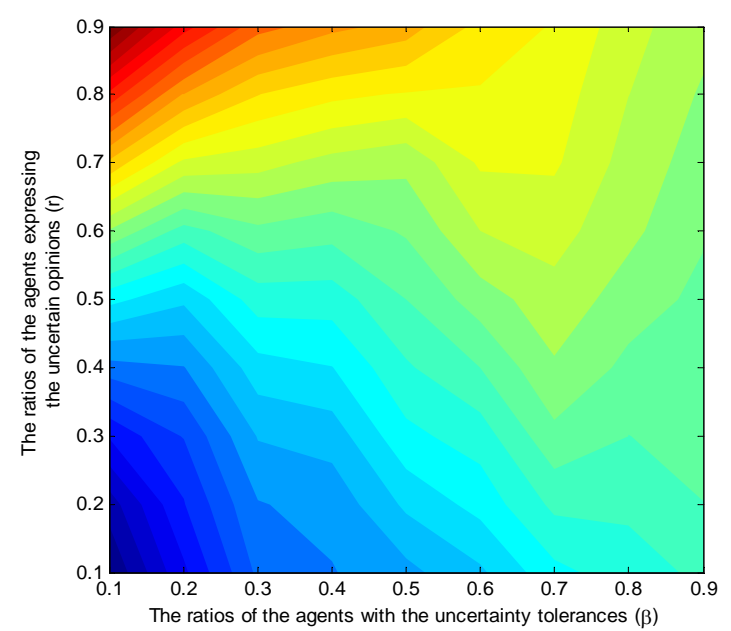

(a)

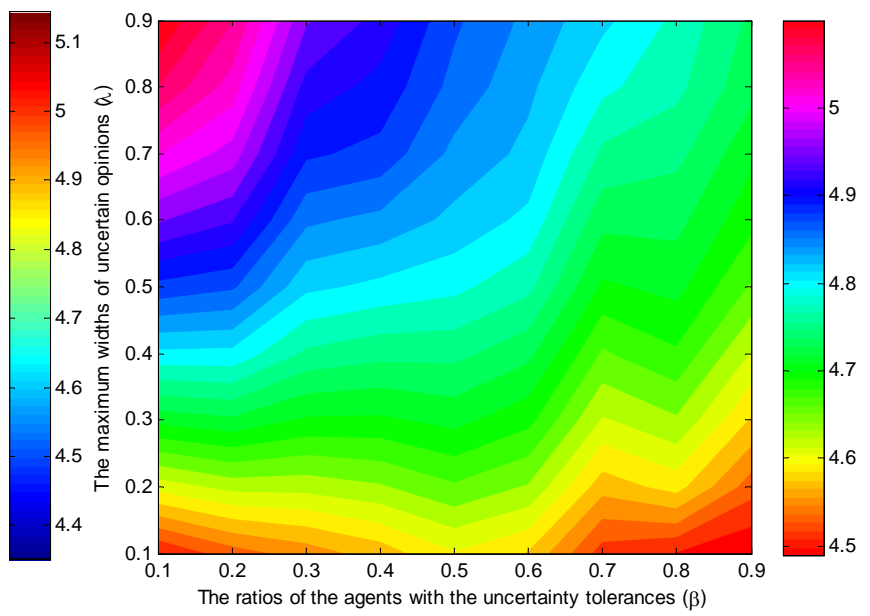

(b)

Figure 7: (a) The average $N C$ values under different $\beta$ and $r$ values; (b) The average $N C$ values under different $\beta$ and $\lambda$ values

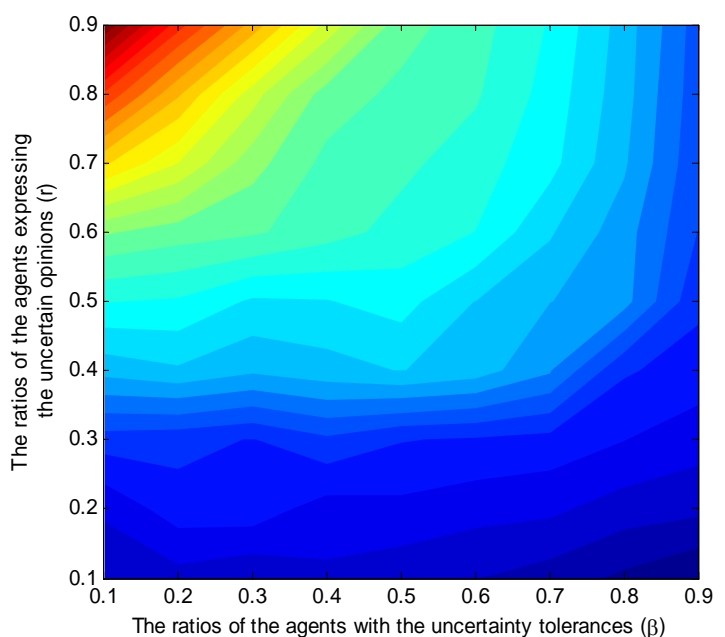

(a)

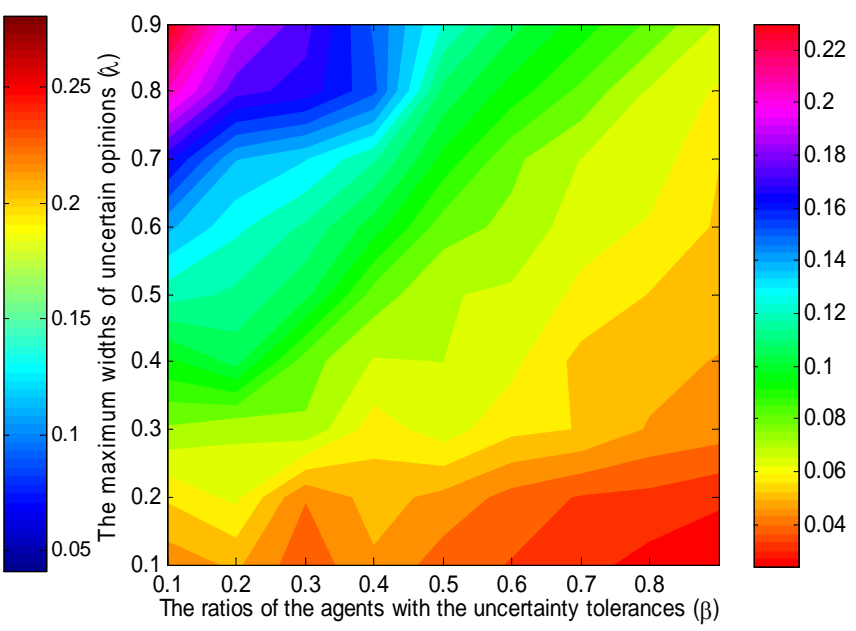

(b)

Figure 8: (a) The average $r_{E S C}$ values under different $\beta$ and $r$ values; (b) The average $r_{E S C}$ values under different $\beta$ and $\lambda$ values

5.3 Figures 7 (a) .7 (b) show the following finding: When $r \geq 0.8$ or $\lambda \geq 0.5$, the average $N C$ values decrease as $\beta$ increases.

The finding implies that when the ratios of the agents expressing uncertain opinions or the widths of uncertain opinions are sufficiently large, the number of clusters will decrease with the increase in the ratios of the agents with the uncertainty tolerances.

The observations from Figures 7(a).7(b) can be explained as follows:

In the simulation, when the ratios of the agent expressing uncertain opinions or the widths of uncertain opinions are sufficiently large, we find that the number of agents in the confidence sets will increase with the increase in the agents with the uncertainty tolerances. Consequently, less clusters will appear.

5.4 Figures 8 (a) -8 (b) show the following finding: When $r \geq 0.7$ or $\lambda \geq 0.4$, the average $r_{E S C}$ values decrease as $\beta$ increases.

This implies that when the ratios of the agents expressing uncertain opinions or the widths of uncertain opinions are sufficiently large, the ratios of the extremely small clusters in all clusters will decrease with the increase in the ratios of the agents with the uncertainty tolerances. The finding in Figure $8(\mathrm{a})-8 \mathrm{~g}$ (b) can be explained as follows: In the simulation, when the ratios of the agent expressing uncertain opinions or the widths of uncertain opinions are sufficiently large, we find that the number of isolated opinions in the dynamics of uncertain opinion formation will decrease with the increase in the ratios of the agents with the uncertainty tolerances. 


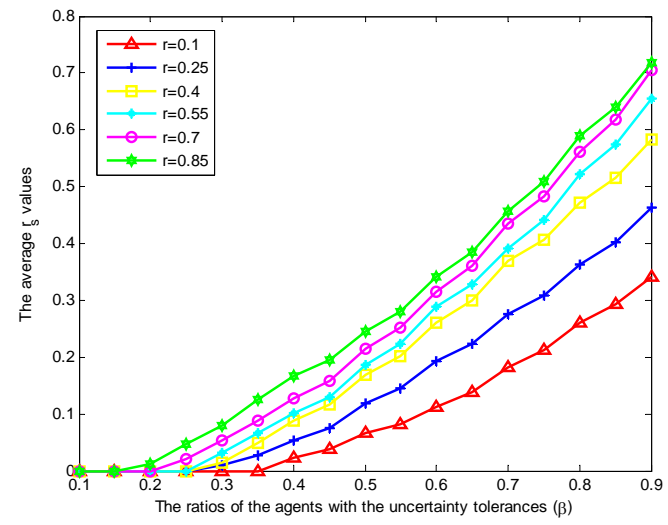

(a)

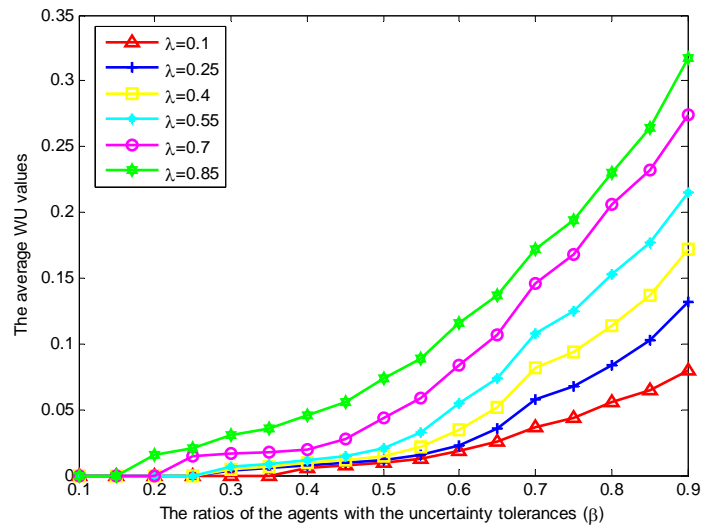

(b)

Figure 9: (a) The average $r_{s}$ values under different $\beta$ and $r$ values; (b) The average $W U$ values under different $\beta$ and $\lambda$ values

Consequently, the smaller ratios of the extremely small clusters in all clusters will appear.

5.5 Figures 9 (a). 9 (b) show the following finding: The average $r_{s}$ and $W U$ values increase as $\beta$ increases. This implies that both the ratios of the agents expressing the uncertain opinions and the average widths of uncertain opinions in the stabilized results will increase with the increase in the ratios of the agents with the uncertainty tolerances. The finding in Figure 9 (a) -9 (b) can be explained as follows: In the simulation, we find that the opinions of the agents without the uncertainty tolerances will gradually become more accurate in the dynamics of uncertain opinion formation. Thus, with the increase in the ratios of the agents with the uncertainty tolerances, the larger ratios of the agents expressing uncertain opinions and the larger average widths of uncertain opinions in the stabilized result will appear.

\section{Conclusions}

6.1 In this study, we investigate the dynamics of uncertain opinion formation based on the bounded confidence model. In the proposed model, the agents express their opinions by using either numerical intervals (i.e., the uncertain opinions) or exact numbers (i.e., the exact opinions). Furthermore, based on different communication regimes, the agents are divided into two types: the agents with uncertainty tolerances and the agents without uncertainty tolerances.

6.2 We use an agent-based simulation to obtain the following findings: (i) With the increase in the ratios of the agents expressing uncertain opinions and the widths of uncertain opinions, more clusters and larger ratios of the extremely small clusters in all clusters will appear. Meanwhile, when the ratios of the agents expressing uncertain opinions or the widths of uncertain opinions are sufficiently large, less clusters and smaller ratios of the extremely small clusters in all clusters will appear with the increase in the ratios of the agents with the uncertainty tolerances; (ii) When all the agents are with the uncertainty tolerances, the ratios of the agents expressing the uncertain opinions in the stabilized result will be larger than those in the initial time. But the average widths of uncertain opinions in the stabilized result will be smaller than those in the initial time; (iii) When there exist certain agents without the uncertainty tolerances, both the ratios of the agents expressing the uncertain opinions and the average widths of uncertain opinions in the stabilized results will be smaller than those in the initial time.

6.3 The proposed model can be applied to address certain opinion formation problems in the real world. For example, when the government attempts to analyse the dynamics of public opinions on introducing a chemical project, certain citizens may express uncertain opinions on the necessity of introducing the chemical project. Furthermore, different citizens who encountered uncertain opinions have different uncertainty tolerances.

6.4 Generally, people express their opinions, sentiments, or support emotions regarding different issues in a social network. However, in this paper, the influences of different social network structures on uncertain opinion formation are not considered. Therefore, it would be an interesting future topic to investigate the dynamics of uncertain opinion formation by considering different social network structures. 


\section{Acknowledgements}

Yucheng Dong would like to acknowledge the financial support of grants (Nos. 71171160, 71571124) from NSF of China, and grants (Nos. skqy201606 and skgt201502) from Sichuan University

\section{Appendix: Model analysis}

In model analysis, we devote to compare the HK model with the proposed model and discuss some desired properties in the proposed model.

Firstly, in the comparative analysis, we show that the proposed model does not satisfy certain properties of the isolated fully connected group presented in the HK model. An isolated fully connected group (Urbig et al. 2008) is a set of agents in which all agents within the same group have a distance smaller than $\varepsilon$, whereas each agent outside the group has a distance larger than $\varepsilon$ to each agent in the group. Here, the distance measure in Eq. (3) is used to define the isolated fully connected group.

Generally, the HK model satisfies four properties as follows:

(i) An isolated fully connected group never splits;

(ii) The mean opinions of the agents in an isolated fully connected group remain stable;

(iii) Different isolated fully connected groups never merge.

In the following, we propose three counterexamples to illustrate that the proposed model does not satisfy (i)(iii).

Example 1 is used to illustrate that (i) is not satisfied in the proposed model. In Example 1, there are four agents $A_{1}, A_{2}, A_{3}, A_{4} \in A^{\circ}$. Their initial opinions are given by

$$
\bar{x}_{i}(0)= \begin{cases}{[0.19,0.3],} & i=1 \\ {[0.2,0.3],} & i=2 \\ {[0,0.1],} & i=3 \\ {[0.02,0.11],} & i=4\end{cases}
$$

We set the bounded confidence as: $\varepsilon=0.2$.

Based on Eq. (4), we find that $d\left(\bar{x}_{i}(t), \bar{x}_{j}(t)\right) \leq \varepsilon$, for $i, j=1,2,3,4$ and $i \neq j$. This indicates that the four agents have formed an isolated fully connected group.

The initial accurate estimations of $A_{1}, A_{2}, A_{3}$ and $A_{4}$ are given as follows:

$A_{1}: f_{12}(0)=0.3, f_{13}(0)=0, f_{14}(0)=0.02 ; A_{2}: f_{21}(0)=0.3, f_{23}(0)=0, f_{24}(0)=0.02$;

$A_{3}: f_{31}(0)=0.3, f_{32}(0)=0.3, f_{34}(0)=0.02 ; A_{4}: f_{41}(0)=0.3, f_{42}(0)=0.3, f_{43}(0)=0$;

Based on Eqs. (8)-(9), we obtain:

$$
\bar{x}_{i}(1)= \begin{cases}{[0.245,0.3],} & i=1 \\ {[0.25,0.3],} & i=2 \\ {[0.01,0.06],} & i=3 \\ {[0.01,0.055],} & i=4\end{cases}
$$

We find: $d\left(\bar{x}_{1}(1), \bar{x}_{2}(1)\right) \leq \varepsilon, d\left(\bar{x}_{3}(1), \bar{x}_{4}(1)\right) \leq \varepsilon$, and $d\left(\bar{x}_{l}(1), \bar{x}_{k}(1)\right)>\varepsilon$, for $l=1,2$ and $k=3,4$. Clearly, the agents $A_{1}$ and $A_{2}$ form an isolated fully connected group at time $t=1$, and the agents $A_{3}$ and $A_{4}$ form the other isolated fully connected group at time $t=1$.

Example $\mathbf{2}$ is used to illustrate that (ii) is not satisfied in the proposed model. In example 2, there are five agents $A_{1}, A_{2}, A_{3}, A_{4}$ and $A_{5}$, where $A_{1}, A_{3}, A_{5} \in A^{u}$ and $A_{2}, A_{4} \in A^{o}$. Their initial opinions are given by

$$
\bar{x}_{i}(0)= \begin{cases}{[0.1,0.3]} & i=1 \\ 0.05, & i=2 \\ 0.25, & i=3 \\ {[0,0.2],} & i=4 \\ 0.1, & i=5\end{cases}
$$

We set the bounded confidence as: $\varepsilon=0.2$.

Based on Eq. (4), we find that $d\left(\bar{x}_{i}(0), \bar{x}_{j}(0)\right) \leq \varepsilon$, for $i, j=1,2, \ldots, 5$ and $i \neq j$. This statement indicates that the five agents have formed an isolated fully connected group. Additionally, the mean opinion (i.e., the average values for $\left.\bar{x}_{i}(0)\right)$ in this isolated fully connected group is equal to $[0.10,0.18]$. 
The initial accurate estimations of agents $A_{2}$ and $A_{4}$ are given as follows:

$A_{2}: f_{21}(0)=0.25, f_{23}(0)=0.25, f_{24}(0)=0.1, f_{25}(0)=0.1$;

$A_{4}: f_{41}(0)=0.2, f_{42}(0)=0.05, f_{43}(0)=0.25, f_{45}(0)=0.1$;

Based on Eqs. (6)-(9), we obtain:

$$
\bar{x}_{i}(1)= \begin{cases}{[0.10,0.18],} & i=1 \\ 0.15, & i=2 \\ {[0.10,0.18],} & i=3 \\ {[0.12,0.16],} & i=4 \\ {[0.10,0.18],} & i=5\end{cases}
$$

We find that $d\left(\bar{x}_{i}(1), \bar{x}_{j}(1)\right) \leq \varepsilon$, for $i, j=1,2, \ldots, 5$ and $i \neq j$. This statement indicates that the five agents remain in an isolated fully connected group. However, the mean opinion at time $t=1$ is equal to [0.114, 0.17].

Example 3 is used to illustrate that (iii) is not satisfied in the proposed model. In Example 3, there are four agents $A_{1}, A_{2}, A_{3}$ and $A_{4}$, where $A_{1}, A_{2}, A_{3}, A_{4} \in A^{o}$. The agents' initial opinions are given by

$$
\bar{x}_{i}(0)= \begin{cases}{[0.245,0.3],} & i=1 \\ {[0.25,0.3],} & i=2 \\ {[0.01,0.06],} & i=3 \\ {[0.01,0.055],} & i=4\end{cases}
$$

and the bounded confidence is assumed as: $\varepsilon=0.2$.

Based on Eq. (4), we find that two isolated fully connected groups exist that are formed at time $t=0$. Specifically, agents $A_{1}$ and $A_{2}$ formed one isolated fully connected group, and agents $A_{3}$ and $A_{4}$ formed the other isolated fully connected group.

The initial accurate estimations of $A_{1}, A_{2}, A_{3}$ and $A_{4}$ are given as follows:

$A_{1}: f_{12}(0)=0.25 ; A_{2}: f_{21}(0)=0.245 ; A_{3}: f_{34}(0)=0.055 ; A_{4}: f_{43}(0)=0.06$;

Based on Eqs. (8)-(9), we obtain:

$$
\bar{x}_{i}(1)= \begin{cases}{[0.2425,0.275],} & i=1 \\ {[0.2425,0.2725],} & i=2 \\ {[0.0325,0.0575],} & i=3 \\ {[0.035,0.0575],} & i=4\end{cases}
$$

Assume that: $f_{12}(t)=f_{21}(t)=0.2425$, and $f_{34}(t)=f_{43}(t)=0.0575$, for $t \geq 1$. Then, based on Eqs. (8) and (9), we obtain:

$$
\bar{x}_{i}(t)=\left\{\begin{array}{ll}
{\left[0.2425, \frac{0.275+0.2425\left(1+2+\cdots 2^{t-1}\right)}{2^{t}}\right],} & i=1 \\
{\left[0.2425, \frac{0.2725+0.2425\left(1+2+\cdots 2^{t-1}\right)}{2^{t}}\right],} & i=2 \\
{\left[\frac{0.0325+0.0575\left(1+2+\cdots 2^{t-1}\right)}{2^{t}}, 0.0575\right],} & i=3 \\
{\left[\frac{0.035+0.0575\left(1+2+\cdots 2^{t-1}\right)}{2^{t}}, 0.0575\right],} & i=4
\end{array} \quad, t \geq 2 .\right.
$$

Because $\lim _{t \rightarrow \infty} \frac{0.275+0.2425\left(1+2+\cdots 2^{t-1}\right)}{2^{t}}=\frac{0.275+0.2425\left(2^{t}-1\right)}{2^{t}}=0.2425$, then we obtain: $\lim _{t \rightarrow \infty} x_{1}(t)=0.2425$. Similarly, we have: $\lim _{t \rightarrow \infty} x_{2}(t)=0.2425$ and $\lim _{t \rightarrow \infty} x_{3}(t)=\lim _{t \rightarrow \infty} x_{4}(t)=0.0575$.

Assume that $x_{1}\left(t^{\prime}\right)=x_{2}\left(t^{\prime}\right)=0.2425$ and $x_{3}\left(t^{\prime}\right)=x_{4}\left(t^{\prime}\right)=0.0575$, Because $\varepsilon=0.2$ and $d\left(\bar{x}_{i}\left(t^{\prime}\right), \bar{x}_{j}\left(t^{\prime}\right)\right) \leq \varepsilon$, for $i, j=1,2,3,4$ and $i \neq j$, then the four agents $A_{1}, A_{2}, A_{3}$ and $A_{4}$ formed an isolated fully connected group at time $t^{\prime}$.

Then, two desired properties in the proposed model are discussed. The first property (see Property 1) indicates that the opinions of the agents without uncertainty tolerances will gradually become more accurate in the dynamics of uncertain opinion formation. The second property (see Property 2 ) indicates that all of the agents will hold an exact opinion when a consensus among the agents is achieved. The two desired properties are provided as follows:

Property 1. Let $\bar{x}_{i}(t)=\left[\bar{x}_{i}^{L}(t), \bar{x}_{i}^{U}(t)\right]$ be as defined previously, and let $d_{i}(t)$ be the width of the opinion $\bar{x}_{i}(t)$, where $d_{i}(t)=\bar{x}_{i}^{U}(t)-\bar{x}_{i}^{L}(t)$. If agent $A_{i} \in A^{o}, d_{i}(t+1) \leq d_{i}(t)$, for $t=0,1, \ldots$;

Proof. Without loss of generality, we assume that $A_{i} \in A^{o}$. Based on Eqs. (7)-(8), if $A_{i}$ expresses the initial opinions using an exact number, $A_{i}$ will continue expressing the exact opinions at any time. If $A_{i}$ expresses the uncertain opinion $\left[\bar{x}_{i}^{L}(t), \bar{x}_{i}^{U}(t)\right]\left(\bar{x}_{i}^{U}(t)>\bar{x}_{i}^{L}(t)\right)$ at time $t$, 
$\bar{x}_{i}^{U}(t+1)-\bar{x}_{i}^{L}(t+1)=w_{i i}(t) \bar{x}_{i}^{U}(t)+\sum_{A_{j} \in \tilde{I}\left(A_{i}, \bar{X}(t)\right), j \neq i} w_{i j}(t) f_{i j}(t)-w_{i i}(t) \bar{x}_{i}^{L}(t)-\sum_{A_{j} \in \tilde{I}\left(A_{i}, \bar{X}(t)\right), j \neq i} w_{i j}(t) f_{i j}(t)$ $=w_{i i}(t)\left(\bar{x}_{i}^{U}(t)-\bar{x}_{i}^{L}(t)\right)$.

Because $0 \leq w_{i i}(t) \leq 1$, then $\bar{x}_{i}^{U}(t+1)-\bar{x}_{i}^{L}(t+1) \leq \bar{x}_{i}^{U}(t)-\bar{x}_{i}^{L}(t)$. Clearly, $d_{i}(t+1) \leq d_{i}(t)$.

This completes the proof.

Property 2. Let $\bar{x}_{i}(t)=\left[\bar{x}_{i}^{L}(t), \bar{x}_{i}^{U}(t)\right]$ and $d_{i}(t)$ be as defined previously. Assume that a consensus among the agents is achieved at time $t$. Then, $d_{i}(t)=0$, for $i=1,2, \ldots, N$.

Proof. Let $\left[\bar{x}_{c}^{L}, \bar{x}_{c}^{U}\right]$ be the uniform opinion of all of the agents at time $t$. Let $A_{i}$ and $A_{j}$ be any two agents, where $A_{i} \in A^{o}$ and $A_{j} \in A^{u}$. Based on Eqs. (6)-(9), we obtain:

$\bar{x}_{i}^{L}(t+1)=\frac{1}{N} \bar{x}_{c}^{L}+\sum_{A_{j} \in \tilde{I}\left(A_{i}, \bar{X}(t)\right), j \neq i} \frac{f_{i j}(t)}{N}, \bar{x}_{i}^{U}(t+1)=\frac{1}{N} \bar{x}_{c}^{U}+\sum_{A_{j} \in \tilde{I}\left(A_{i}, \bar{X}(t)\right), j \neq i} \frac{f_{i j}(t)}{N}$.

and

$\bar{x}_{j}^{L}(t+1)=\frac{1}{N} \bar{x}_{c}^{L}+\sum_{A_{j} \in \tilde{I}\left(A_{i}, \bar{X}(t)\right), j \neq i} \frac{\bar{x}_{c}^{L}}{N}=\bar{x}_{c}^{L}, \bar{x}_{j}^{U}(t+1)=\frac{1}{N} \bar{x}_{c}^{U}+\sum_{A_{j} \in \tilde{I}\left(A_{i}, \bar{X}(t)\right), j \neq i} \frac{\bar{x}_{c}^{U}}{N}=\bar{x}_{c}^{U}$.

Because the consensus among all of the agents remains stable, then:

$\bar{x}_{i}^{L}(t+1)=\bar{x}_{j}^{L}(t+1)=\bar{x}_{c}^{L}$ and $\bar{x}_{i}^{U}(t+1)=\bar{x}_{j}^{U}(t+1)=\bar{x}_{c}^{U}$.

Furthermore, we have: $\frac{f_{i j}(t)}{N}=\frac{\bar{x}_{c}^{L}}{N}=\frac{\bar{x}_{c}^{U}}{N}$. As a result, $f_{i j}(t)=\bar{x}_{c}^{L}=\bar{x}_{c}^{U}$.

This completes the proof.

\section{References}

Afshar, M. \& Asadpour, M. (2010). Opinion formation by informed agents. Journal of Artificial Societies and Social Simulation, 13(4)

Blondel, V. D., Hendrickx, J. M. \& Tsitsiklis, J. N. (2009). On krause's multi-agent consensus model with statedependent connectivity. IEEE transactions on Automatic Control, 54(11), 2586-2597

Ceragioli, F. \& Frasca, P. (2012). Continuous and discontinuous opinion dynamics with bounded confidence. Nonlinear Analysis: Real World Applications, 13(3), 1239-1251

Deffuant, G., Carletti, T. \& Huet, S. (2013). The leviathan model: Absolute dominance, generalised distrust, small worlds and other patterns emerging from combining vanity with opinion propagation. Journal of Artificial Societies and Social Simulation, 16(1)

Deffuant, G., Neau, D., Amblard, F. \& Weisbuch, G. (2000). Mixing beliefs among interacting agents. Advances in Complex Systems, 3(01n04), 87-98

DeGroot, M. H. (1974). Reaching a consensus. Journal of the American Statistical Association, 69(345), 118-121

Dong, Y. \& Herrera-Viedma, E. (2015). Consistency-driven automatic methodology to set interval numerical scales of 2-tuple linguistic term sets and its use in the linguistic gdm with preference relation. IEEE transactions on cybernetics, 45(4), 780-792

Dong, Y., Zhang, G., Hong, W.-C. \& Yu, S. (2013). Linguistic computational model based on 2-tuples and intervals. IEEE Transactions on fuzzy systems, 21(6), 1006-1018

Etesami, S. R., Başar, T., Nedić, A. \& Touri, B. (2013). Termination time of multidimensional hegselmann-krause opinion dynamics. In 2013 American Control Conference, (pp. 1255-1260). IEEE

Fortunato, S., Latora, V., Pluchino, A. \& Rapisarda, A. (2005). Vector opinion dynamics in a bounded confidence consensus model. International Journal of Modern Physics C, 16(10), 1535-1551

French Jr, J. R. (1956). A formal theory of social power. Psychological review, 63(3), 181

Friedkin, N. E. \& Johnsen, E. C. (1990). Social influence and opinions. Journal of Mathematical Sociology, 15(3-4), 193-206 
Hegselmann, R., König, S., Kurz, S., Niemann, C. \& Rambau, J. (2015). Optimal opinion control: The campaign problem. Journal of Artificial Societies and Social Simulation, 18(3)

Hegselmann, R., Krause, U. et al. (2002). Opinion dynamics and bounded confidence models, analysis, and simulation. Journal of Artificial Societies and Social Simulation, 5(3)

Krapivsky, P. \& Redner, S. (2003). Dynamics of majority rule in two-state interacting spin systems. Physical Review Letters, 90(23), 238701

Laguna, M., Abramson, G. \& Zanette, D. H. (2003). Vector opinion dynamics in a model for social influence. Physica A: Statistical Mechanics and its Applications, 329(3), 459-472

Latané, B. (1981). The psychology of social impact. American psychologist, 36(4), 343

Lim, D., Lee, H., Zo, H. \& Ciganek, A. (2014). Opinion formation in the digital divide. Journal of Artificial Societies and Social Simulation, 17(1), 13

Lorenz, J. (2005). A stabilization theorem for dynamics of continuous opinions. Physica A: Statistical Mechanics and its Applications, 355(1), 217-223

Mckeown, G. \& Sheehy, N. (2006). Mass media and polarisation processes in the bounded confidence model of opinion dynamics. Journal of Artificial Societies and Social Simulation, 9(1)

Morarescu, I.-C. \& Girard, A. (2011). Opinion dynamics with decaying confidence: Application to community detection in graphs. IEEE Transactions on Automatic Control, 56(8), 1862-1873

Pineda, M., Toral, R. \& Hernandez-Garcia, E. (2009). Noisy continuous-opinion dynamics. Journal of Statistical Mechanics: Theory and Experiment, 2009(08), P08001

Pineda, M., Toral, R. \& Hernández-García, E. (2013). The noisy hegselmann-krause model for opinion dynamics. The European Physical Journal B, 86(12), 1-10

Righi, S. \& Carletti, T. (2009). The influence of social network topology in a opinion dynamics model. In Proceeding of the European Conference on Complex systems

Salzarulo, L. (2006). A continuous opinion dynamics model based on the principle of meta-contrast. Journal of Artificial Societies and Social Simulation, 9(1)

Sutton, J. L., Cosenzo, K. A. \& Pierce, L. G. (2004). Influence of culture and personality on determinants of cognitive processes under conditions of uncertainty. Tech. rep., DTIC Document

Urbig, D., Lorenz, J. \& Herzberg, H. (2008). Opinion dynamics: The effect of the number of peers met at once. Journal of Artificial Societies and Social Simulation, 11(2)

Wang, H. \& Shang, L. (2015). Opinion dynamics in networks with common-neighbors-based connections. Physica A: Statistical Mechanics and its Applications, 421, 180-186

Weisbuch, G. (2004). Bounded confidence and social networks. The European Physical Journal B-Condensed Matter and Complex Systems, 38(2), 339-343 